\title{
VITRAN: an Advance Statistic Tool to Evaluate Fretting-wear Damage*
}

\author{
Pablo R. RUBIOLO** and Michael Y. YOUNG*** \\ ** New Reactor Fuel Engineering Dept., Westinghouse Electric Company, LLC \\ 4350 Northern Pike, Monroeville, PA 15146, USA \\ E-mail: rubiolpr@westinghouse.com \\ *** Fuel Engineering, Westinghouse Electric Company, LLC \\ 5801 Bluff Road, Columbia, SC 29250, USA
}

\begin{abstract}
A non-linear vibration model (called VITRAN for VIbration TRansient Analysis Nonlinear) of the dynamic response of a nuclear fuel rod and its supports has been developed and integrated to a fretting-wear analysis method to predict the performance of fuel assemblies. The approach includes the hydraulic, structural and tribological effects considered to be of sufficient importance. A general description of the software and an example of application are provided in this paper.
\end{abstract}

Key words: Flow-Induced Vibration, Fretting-Wear, Fuel Assemblies

\section{Introduction}

A non-linear vibration model (called VITRAN for VIbration TRansient Analysis Nonlinear) of the dynamic response of a nuclear fuel rod and its supports has been developed and integrated to a fretting-wear analysis method to predict the performance of fuel assemblies ${ }^{(1)}$. The approach includes the hydraulic, structural and tribological effects considered to be of sufficient importance. VITRAN has a modular software organization to optimize the maintenance and robustness, and to reduce the development risk. One of the applications of VITRAN is the prediction of scoping fretting-wear endurance tests of fuel assemblies. These experiments are performed in the Westinghouse Vibration Investigation and Pressure-drop Experimental Research (VIPER) loop. This facility enables testing up to two full fuel assemblies under temperature, pressure and turbulent flow conditions which are considered representative of the reactor core environment. VITRAN has already been successfully used to model these tests and is currently providing guidance in selecting rod support designs, assessing the effect of changes to current designs, and implementing margin improvement measures ${ }^{(2)}$. A general description of the model capabilities and software organization is provided in the following sections.

\section{Non-Linear Vibration Model}

VITRAN takes into account the presence of rod-to-grid gaps caused by cladding creepdown and grid growth. It calculates the rod frequency response and motion, the support impact forces, the work and wear rates, and the depths of the resulting wear scars. The fuel rod model was developed by approximating the rod as an Euler-Bernoulli beam. The assembly grids are modeled as constraining forces that are activated anytime the rod

*Received 12 Sep., 2007 (No. 07-0545) [DOI: 10.1299/.jpes.2.57] 
reaches the limit of the available clearance inside the cells of the grids. An example of a six mid-grids fuel rod model is shown in Fig. 1.

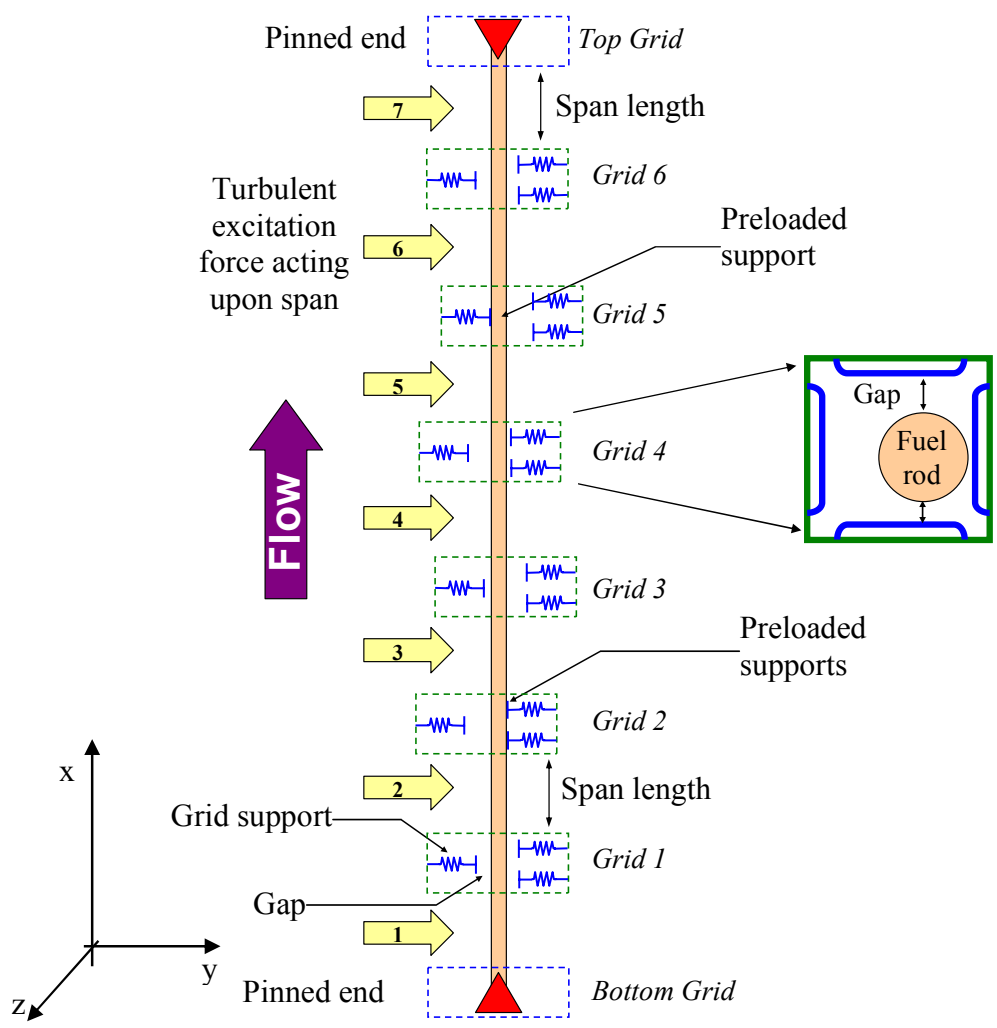

Fig. 1 VITRAN's model for a six mid-grids fuel rod.

The support impact forces are considered as point contact forces and decomposed into: (1) Normal impact force and (2) Tangential or friction force. The non-linearities associated to the grid forces are handled as generalized pseudo-forces ${ }^{(3)}$. The normal impact force is calculated by modeling each support as a spring-damper system with a gap as shown in Fig. 2. Depending on the rod initial conditions, a support could have an initial preload or gap. During the transient simulation and as a result of external forces acting on the rod (e.g. turbulence force) the rod may impact against the gapped supports ${ }^{(4)}$.

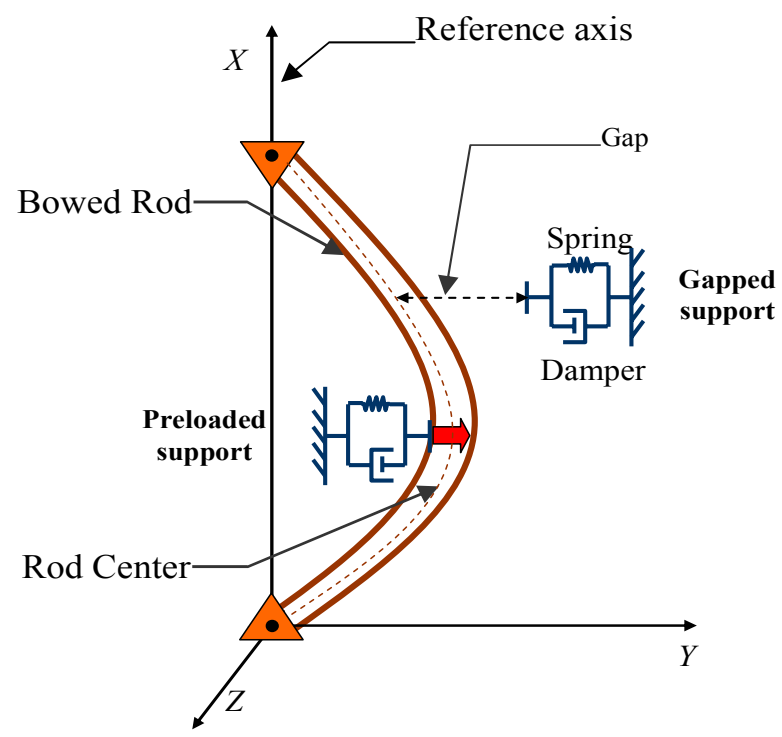

Fig. 2 The normal forces during impacts are calculated using a spring-damper system. 
Two friction models are available for calculating the support tangential force during the impacts: (1)- Velocity Limited Friction Model (VLFM) and (2)- Spring-Damper Friction Model (SDFM). The VLFM calculates the friction force using a classic Coulomb law when the relative velocity between the rod and the support are not close to zero. In the vicinity of zero velocity the Coulomb model is replaced by a viscous force. The SDFM is a more sophisticate model since it defines sliding and sticking regimes for the duration of the impacts. During sliding, the SDFM uses a Coulomb model to calculate the friction force. However, when a zero-cross condition is detected in the tangential velocity (between the rod and the support), the SDFM creates a bound between the two surfaces. The bound is composed of a spring-damper system that simulates the adherence force. The characteristic values of the adherence spring-damper system are set high enough to rapidly reduce the relative motion between the surfaces. The process of setting-up the spring-damper bound is sketched in Fig. 3. The tangential displacements during the sticking regime are negligible with respect to those occurring during sliding motion. When the adherence force exceeds the static friction limit, the SDFM breaks up the bound and switch back to sliding motion (i.e. the Coulomb model). For simulations involving fuel assemblies in the core or tested in VIPER loop, the SDFM provides better accuracy since it correctly predicts the adherence under high preload and the resulting reduction of wear damage.

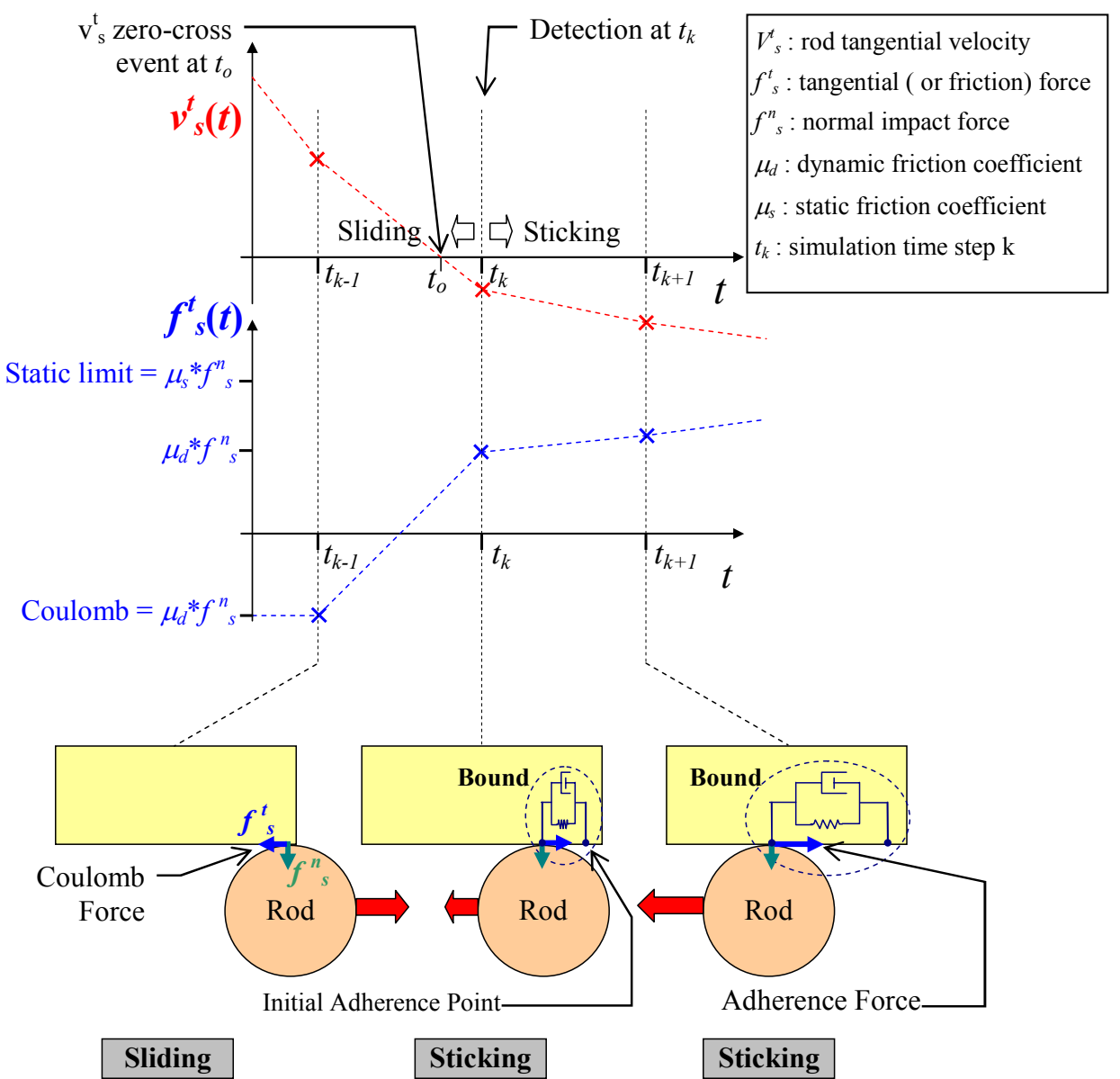

Fig. 3 Process of setting up a Spring-Damper to model the adherence during sticking regime.

VITRAN can model different external forces, including electromechanical shaker excitation forces (in a testing facility) and forces arising from turbulent flow in the fuel bundle (reactor core or VIPER). The turbulence forces are generally approximated as Gaussian Band-Limited White Noise (GBLWN) signals distributed along the rod and with specific correlation domains (see Fig. 4). The GBLWN is a random Gaussian signal with a 
constant Power Spectral Density (PSD) between lower and upper cut-off frequencies. The correlated domains are defined as the sections of the rod where the force is correlated over time, i.e. has the same time variation.

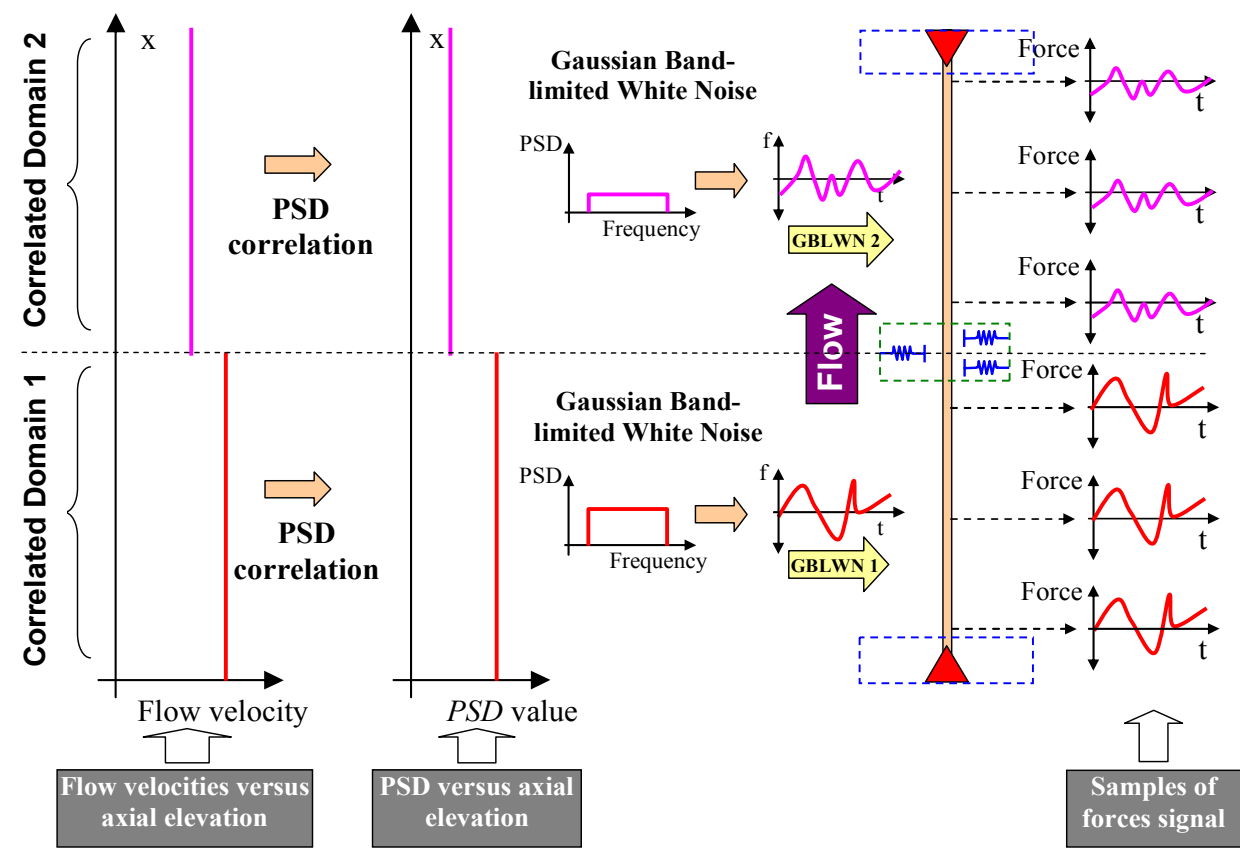

Fig. 4 Example of the steps required to setup the turbulence force in VITRAN.

In most applications, the correlated domains are taken equal to the spans between the grids. The turbulence PSD is then estimated from an experimental correlation developed for axial turbulent flow in fuel bundles. The correlation is a function of the system properties such as the rod diameter and pitch, the fluid properties, the correlation length and the averaged flow velocity in each correlated domains (usually the assembly spans). The flow velocities are determined from a T\&H sub-channel model, such as the one shown in Fig. 5, developed in VIPRE-W. An example of the steps involved in setting up the turbulence forces in VITRAN is illustrated in Fig. 4. For simplicity the example considers only one mid-grid, the correlated lengths are taken equal to the two spans between the grid and the rod ends, and the flow velocity is considered uniform over each span.

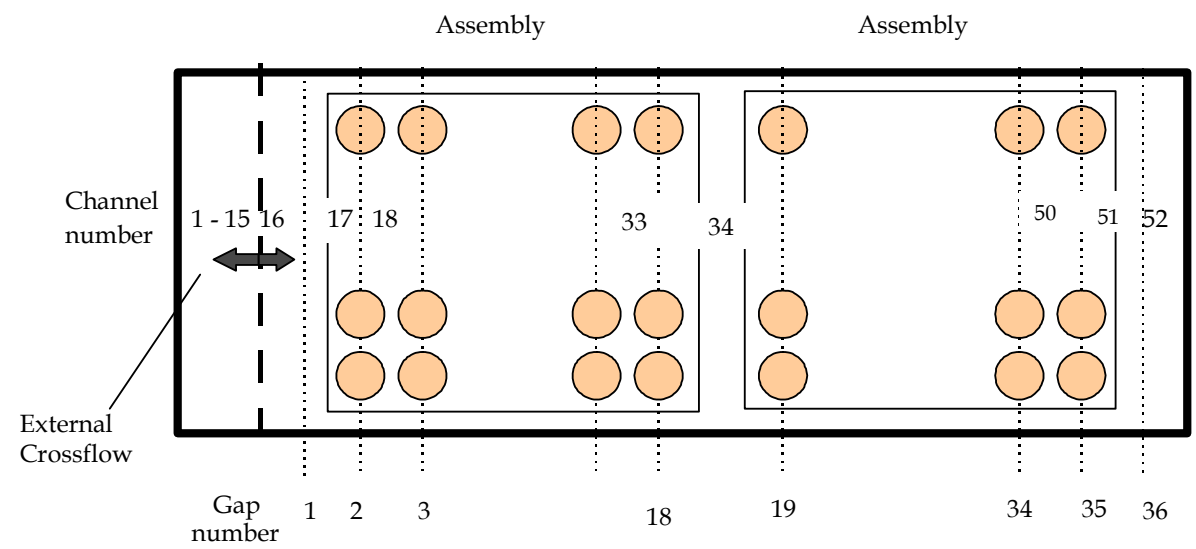

Fig. 5 VIPRE-W model of two side-by-side fuel assemblies in VIPER test loop.

Once the mechanical properties, boundary and initial conditions of the fuel rod, the flow velocities and the external forces have been defined, a single case simulation can be performed using the Fuel Rod Simulator (FRS). The FRS is the core application of VITRAN and consists of an Ordinary Differential Equation (ODE) solver that integrates the 
motion equations during a prescribed transient time. As shown in Fig. 6, the Fuel Rod Simulator handles the non-linear term associated to the impact forces by considering the support forces as generalized pseudo-forces ${ }^{(3)}$. Hence their values are iteratively computed during the numerical resolution (similarly to the external force term) and according to the rod position rather than being analytically solved. This operation allows using the unconstrained rod vibration modes to expand the rod displacements. The rod averaged displacements parameters and the normal impact forces are later determined from the fuel rod transient simulation, and used to calculate the normal work rates at the grids ${ }^{(5)}$. Finally by using the Archard's law, the rod-to-grid wear coefficient and the geometric characteristics of the worn surfaces, VITRAN estimates the volumetric wear rates, the wear volumes and the resulting scars depths.

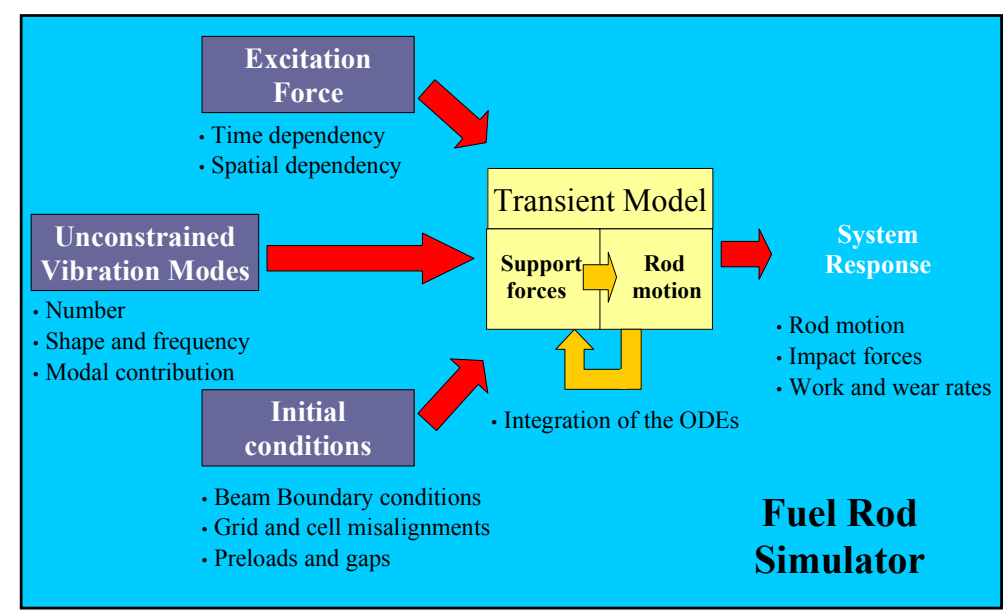

Fig. 6 VITRAN Fuel Rod Simulator (FRS).

\section{Statistic Analysis}

A single transient simulation is only appropriate to predict the fretting-wear performance of a fuel rod in high controlled experimental conditions where most of the parameters are accurately known. On the contrary, the prediction of the performance of rods in fuel assemblies tested in VIPER loop or operated in the reactor core requires the use of statistic analysis tools because of the uncertainties on the fuel rod boundary conditions and mechanical properties. These uncertainties have different causes including the core coolant flow conditions, the irradiation and temperature fields and, in a lesser degree, the manufacturing tolerances. Fig. 7 illustrates, as an example, the variability that can be observed in the wear coefficient.

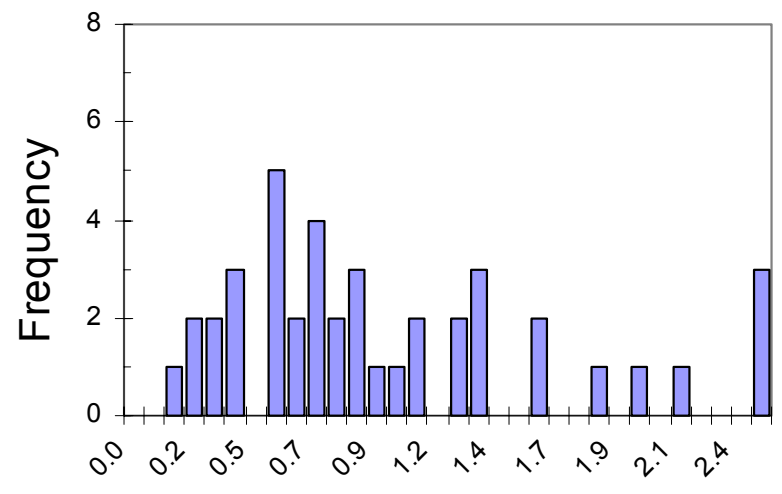

Normalized Wear Coefficient

Fig. 7 Experimentally measured wear coefficient for a typical grid support. 
The statistic analysis is implemented in VITRAN by using a Monte Carlo (MC) method according to the following steps ${ }^{(6)}$ :

- Define the random variables parameters

- Generate input cases by sampling the random variables according to their Probability Density Functions (PDFs)

- Perform the non-linear vibration transient simulation and store the results

- Statistical analysis of the results

The following parameters have to be defined prior to the MC simulation: (a) Fuel assembly variables to be randomized, (b) Probability distributions and Standard Deviations (STD) of the random variables, and (c) Total number of samples. The mean values of the random variables are defined in a reference case (Single Case Input File). VITRAN supports the following probability distributions: (1) Exponential, (2) Extreme Value, (3) Lognormal, (4) Normal, (5) Rayleigh and (6) Uniform. Minimum and maximum values can also be provided to define data rejection criterion when generating the samples of the random variables.

\section{Software Organization}

VITRAN software has a modular organization in order to:

- Optimize maintenance \& robustness: all components have defined functions and interfaces between each other; and

- Reduce development risk: failure to achieve adequate performance of a module has a limited impact on the overall software.

The program is composed by six modules as shown in Fig. 8: (1)- Fuel Rod Simulator containing the non-linear vibration transient model of the fuel rod, (2)-(3) Pre- and Post-processors for data analysis of single case simulations, (4)- Statistic Analysis Module to setup Monte Carlo analyses, and (5)-(6) Statistic Analysis Pre- and Post-processors.

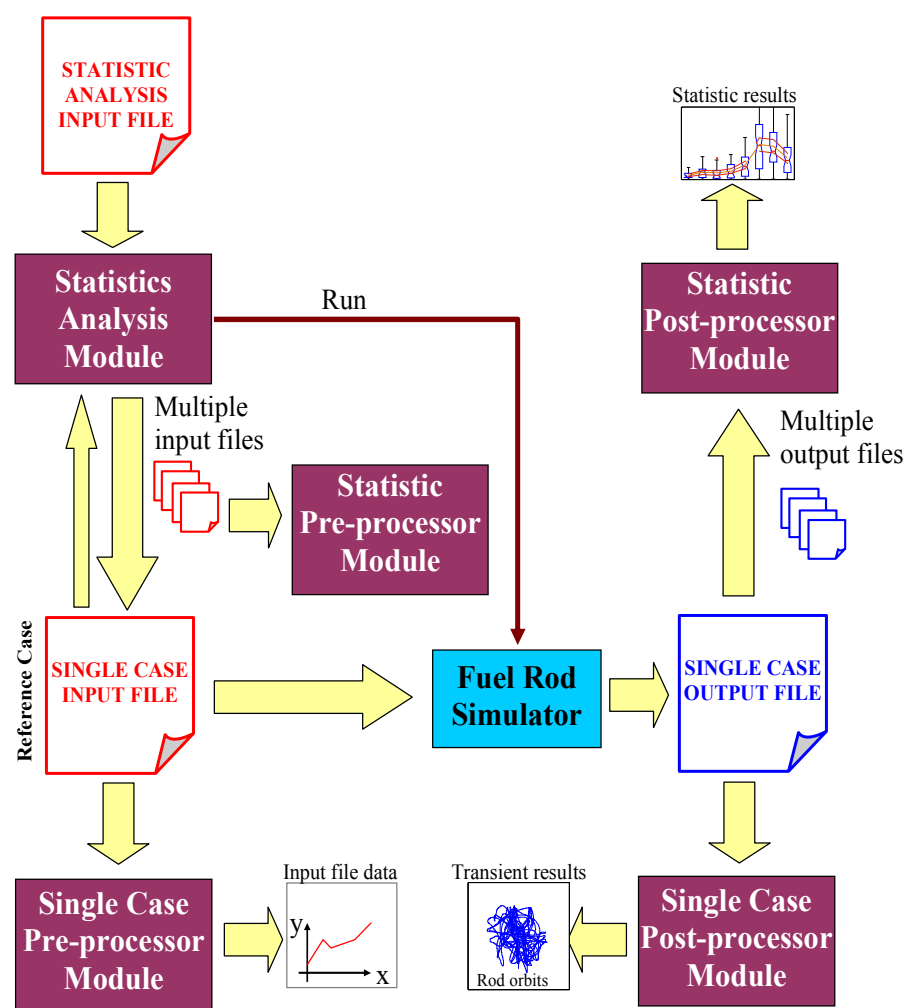

Fig. 8 VITRAN software organization. 
The Statistic Analysis Module generates the Monte Carlo samples according to the distribution parameters defined in the Statistic Analysis Input File (defining the number of sample cases, the name of the random variables, the distribution types and STD) and the mean values defined in a reference case. The Statistic Analysis Module stores the sample cases in a special folder and upon User request, loads each sample and calls the Fuel Rod Simulator to perform a transient simulation. At the end of each simulation, the results are saved into a folder containing a set of Single Case Output Files. Once the transient simulations are completed, a Statistic Analysis Post-processor reads the output files and calculates average values, standard deviations and other statistical parameters of the probability density functions of the wear/work rates and the wear scar population. VITRAN also contains a Statistic Analysis Pre-processor that can be used to verify that adequate statistic is obtained with the sample cases.

\section{Data Post-processing}

VITRAN's pre- and post-processors functions are accessed through Graphic User Interfaces (GUIs) designed to simplify the analysis and the interpretation of the simulations results. The data plots are interactive allowing the User to perform detailed analysis including data fitting, interpolation and statistics. Results of the simulations can also be output as ASCII files.

\subsection{Single Case Simulation}

The Single Case Post-processor performs data analysis using the information stored in a Single Case Output File. The outputs from this module include:

(1) Rod motion data: time averaged displacements and accelerations (mean, RMS, etc), displacement and acceleration spectrums and rod orbits (at any rod elevation).

(2) Individual support data: wear and work rates, scar average depths, averaged sliding time and distance, averaged sticking time and distance, number of impacts and averaged impact forces in each grid support.

(3) Grid data (considering all supports in the cell): wear and work rates values averaged over the grid supports. Rod displacements inside the fuel rod grids.

The Single Case Post-processor also provides information regarding the input data such as the initial conditions (preload and gaps) and the excitation forces. Upon User request, this post-processor can also output dynamic simulation data for any particular support in the form of a panel containing the rod orbits, displacements, velocities and impact forces (see Fig. 9). These plots are interactive and particularly useful to evaluate the performance and stability of the friction model. Although the Single Case Post-processor is usually used to analyze single case simulations, it can also be employed to investigate any potential outlier detected in the Monte Carlo simulation. 


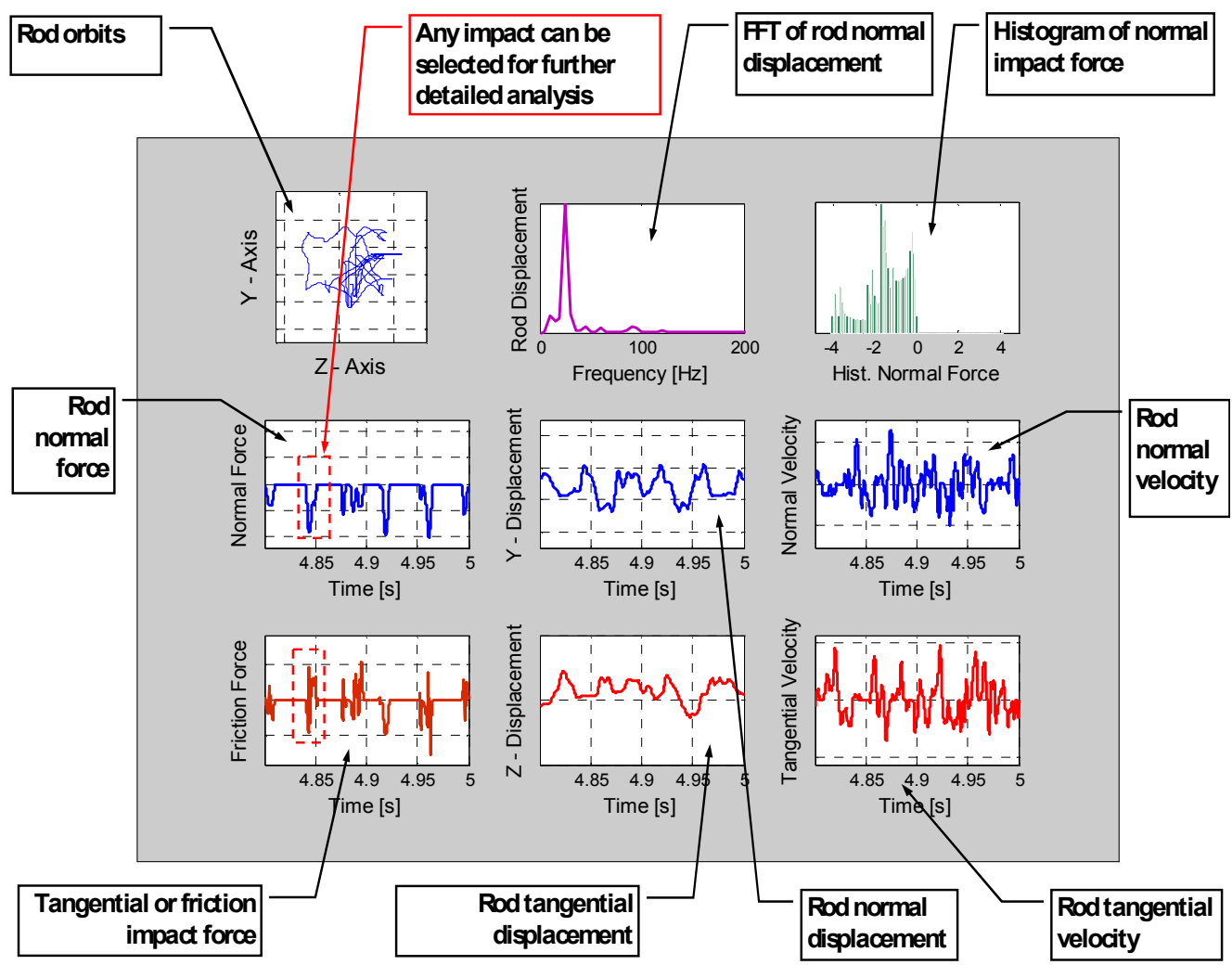

Fig. 9 Rod-support detailed information included plots for the normal and friction forces, support force histogram, rod normal and tangential displacements, rod normal and tangential velocities, PSD of the rod displacements and orbits (at the support axial elevation)

\subsection{Statistic Analysis}

VITRAN Statistic Analysis Post-processor scans the folder containing the Monte Carlo output files and calculates the statistics properties of the parameters of interest. The statistic data for the individual grid supports included:

- Mean values, standard deviation, minimum and maximum values of the wear and work rates, scars depths, sliding and sticking distances and time. Average number of impacts, impact forces and number of scars.

- Probability density functions of any of the above parameters.

The statistic data for the grids included:

- Number of contacted and worn cell sides.

- Mean values, standard deviation, mean lower bound, mean upper bound and maximum values of the scar depths, wear rate and work rate in the grid.

VITRAN Statistic Post-processor enables various types of advance analyses, including multivariate statistics and distribution data fitting. Multivariate statistics is particularly useful in the present applications since it allows visualizing data that has many possible variables and can be used to identify relationships between the wear parameters as shown in the example of Fig. 10. In this case the data was grouped according to the support type: spring and dimples (other grouping criteria can also be defined). The output formats for the grid statistic data are also optimized to allow for an easy comparison against data collected in VIPER test or from irradiated fuel assemblies. For example Fig. 11 illustrates the grid volumetric wear rate averaged over the total number of cells predicted by VITRAN and the values measured in VIPER test. The postprocessor also allows to group data according to 
the grid support type. For example, Fig. 12 presents the predicted volumetric wear rate in the same set of simulations for spring and dimples. This plot allows comparing the relative fretting-wear margin of these two supports.

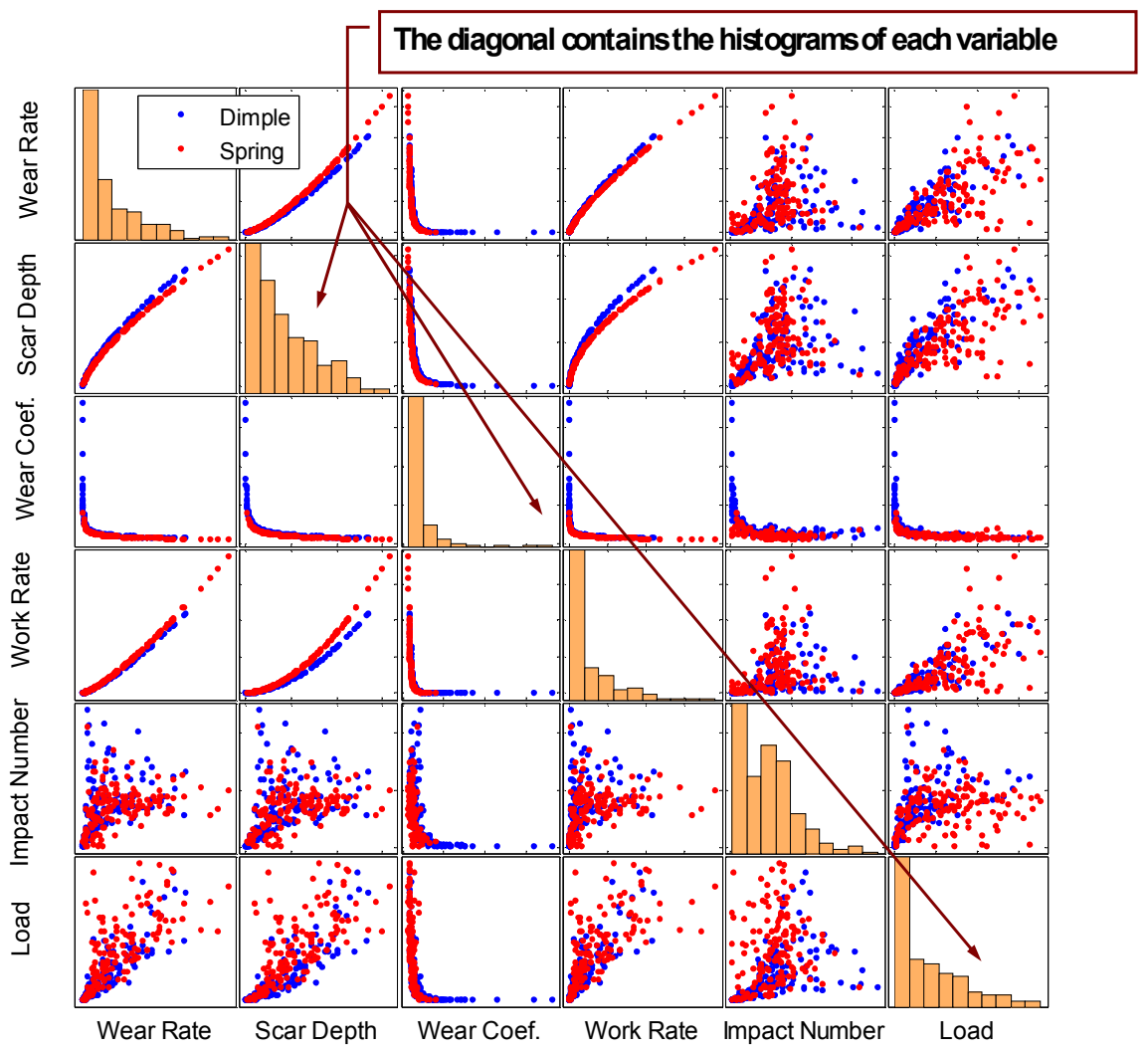

Fig. 10 Multivariate analysis of support data using the support type as grouping criterion.

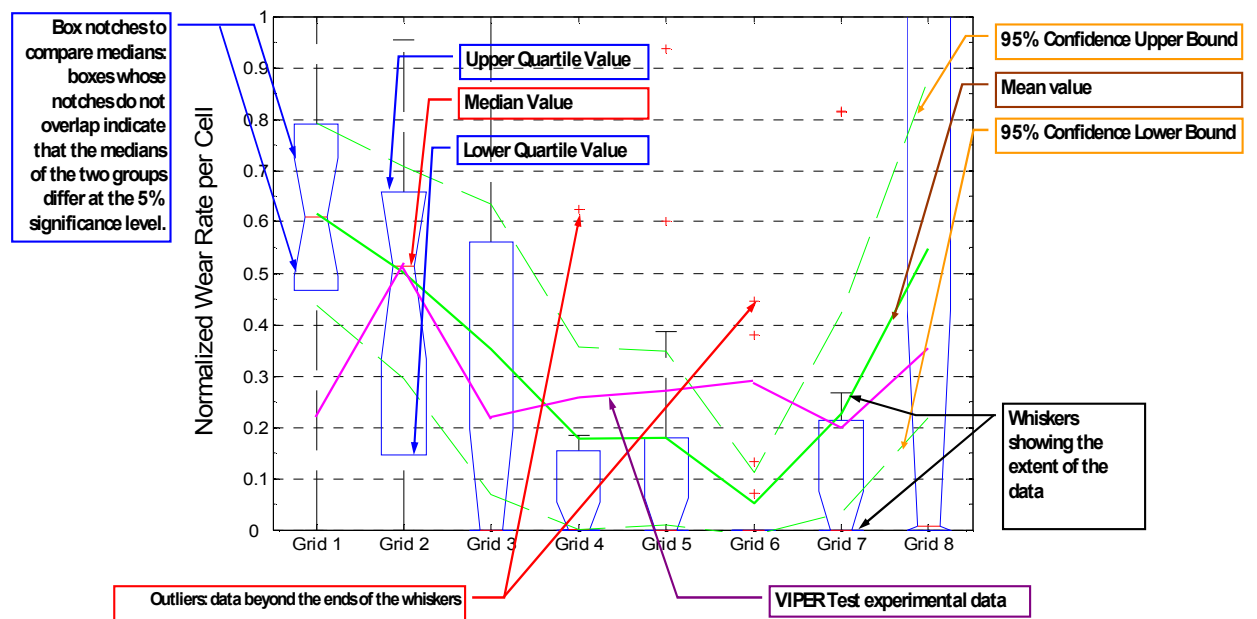

Fig. 11 Grid statistic: volumetric wear rate average over the total number of cells. VITRAN predictions and VIPER experimental data are compared. 


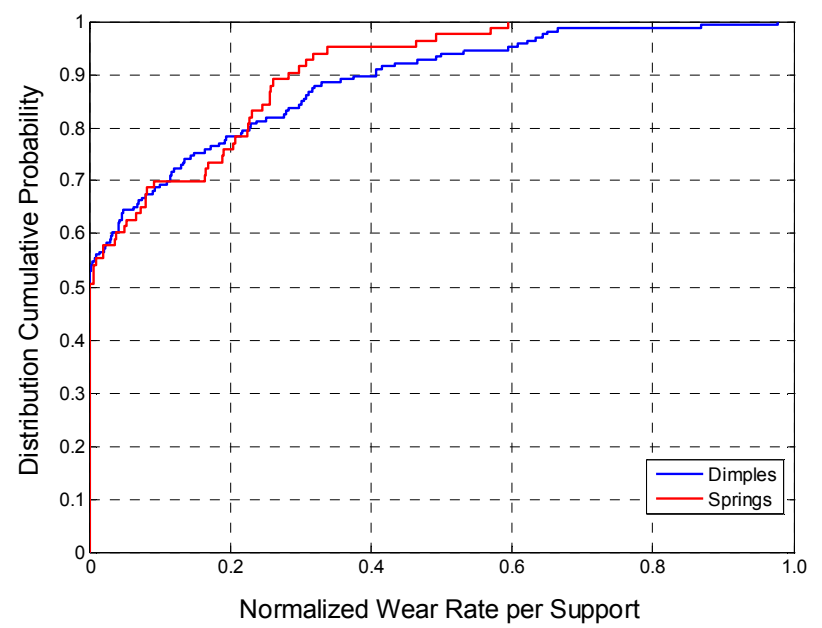

Fig. 12 - Predicted cumulative probability density for the volumetric wear rate for the springs and the dimples.

\section{Summary}

A non-linear vibration model (called VITRAN for VIbration TRansient Analysis Nonlinear) of the dynamic response of a nuclear fuel rod and its supports has been developed and integrated to a fretting-wear analysis method to predict the performance of fuel assemblies. One of the applications of VITRAN is as a prediction tool that helps to reduce the number of scoping fretting-wear endurance tests of fuel assemblies. These experiments are performed in the Westinghouse Vibration Investigation and Pressure-drop Experimental Research (VIPER) loop. VITRAN has been successfully used to predict these tests and is currently providing guidance in selecting rod support designs, assessing the effect of changes to current designs, and implementing margin improvement measures. A general description of the software and its capabilities was provided in this paper.

\section{References}

(1) Rubiolo, P.R., "Probabilistic Prediction of Fretting-wear Damage of Nuclear Fuel Rods", Nuclear Engineering and Design, 236, (2006), pp. 1628-1640.

(2) Rubiolo, P.R., et al., "On the Factors Affecting the Fretting-wear Risk of PWR Fuel Assemblies", Proceedings of the 14th International Conference on Nuclear Engineering (ICONE 14), Miami, July 17-20, (2006), CD-ROM.

(3) Molnar, A.J., et al., "Application of Normal Mode Theory and Pseudoforce Methods to Solve Problems with Nonlinearities", Journal of Pressure Vessel Technology, (1976), pp. 151-156.

(4) Rubiolo, P.R., et al., "Study of Fuel Rod and Grid Supports Interaction", Proceedings of the ASME Symposium on Flow-Induced Vibration (PVP-2003), Cleveland, 2003, CD-ROM.

(5) Frick, T.M., et al., "Overview on the Development and Implementation of Methodologies to Compute Vibration and Wear of Steam Generator Tubes", Proceedings of the ASME Symposium on Flow-Induced Vibration, Vol. 3: Vibration in Heat Exchangers, New Orleans, (1984), pp. 149-161.

(6) Rubiolo, P.R., "Monte Carlo Simulation of Fretting Wear Performance of Fuel Rods", Proceedings of the ASME Symposium on Flow-Induced Vibration (PVP-2005), Denver, (2005), CD-ROM. 